Nonlinear Processes in Geophysics (2005) 12: 163-170

SRef-ID: $1607-7946 / \mathrm{npg} / 2005-12-163$

European Geosciences Union

(c) 2005 Author(s). This work is licensed

under a Creative Commons License.

\title{
Phase synchronization of slip in laboratory slider system
}

\author{
T. Chelidze ${ }^{1,2}$, T. Matcharashvili ${ }^{1}$, J. Gogiashvili ${ }^{1}$, O. Lursmanashvili ${ }^{2}$, and M. Devidze ${ }^{1}$ \\ ${ }^{1}$ Institute of Geophysics of Georgian Academy of Sciences, 1 Alexidze str, 0193, Tbilisi, Georgia \\ ${ }^{2}$ Tbilisi State University, 1 Chavchavadze Ave, Tbilisi, Georgia
}

Received: 9 August 2004 - Revised: 19 November 2004 - Accepted: 6 January 2005 - Published: 31 January 2005

Part of Special Issue "Seismicity pattern dynamics"

\begin{abstract}
In the present study the character of slip regimes in laboratory spring-slider system under weak external periodical forcing has been investigated. We report the experimental evidence of phase synchronization (PS) in a slip dynamics, induced by the external periodic electromagnetic (EM) impact. The quality of synchronization depends on the intensity and frequency of the applied field; the corresponding Arnold's tongue region is constructed. Application of special techniques (measuring phase differences, phase diffusion coefficient, Shannon entropy, Recurrence Quantification Analysis) allows quantitative assessment of the strength of synchronization of microslips with EM impact. It is also shown that the character of power law relationship in acoustic emission amplitude (energy) distribution also undergoes significant changes at changing excitation intensity.
\end{abstract}

\section{Introduction}

It is known that many physical, biological or technical systems often respond in a very original (nonlinear) way to the external driving signals. One of the possible responses is synchronization of a system dynamics by a weak external perturbation. For the last decade synchronization phenomena in complex dynamical systems attracted much interest (Pikovsky et al., 2003). At present various types of synchronization are known, e.g. complete or identical synchronization, generalized synchronization, lag and phase synchronization (Pikovsky et al., 2003; Rosenblum et al., 1996, 1997). In geophysics the study of synchronization phenomenon is connected with such important problem as modification of seismic regime by weak external forces, such as tides, strong electrical (MHD) pulses, magnetic storms, reservoir impounding, etc (Dietrich, 1987; Nikolaev, 1994; Grasso, 1992; Vidale et al., 1998; Tarasov et al., 1999; Custodio et al., 2002; Sobolev and Ponomarev, 2003; Beeler and

Correspondence to: T. Chelidze

(chelidze@ig.acnet.ge)
Lockner, 2003; Perfettini and Schmittbuhl, 2001: Scholz, 2003; Wang et al., 2004). In some cases, as lunar quakes and volcanic tremors (Custodio et al., 2002) the tidal effect is very clear. At the same time, the correlation of tectonic earthquakes with a weak external forcing is still questionable (Vidale et al., 1998; Beeler and Lockner, 2003). That is why we tried to study the synchronization effect in laboratory slider-spring system under controllable conditions; it is believed that this system mimics seismic process, namely, shear displacement on active faults (Rundle et al., 2000).

The experiments on laboratory spring-slider system subjected to a constant pull with the superimposed weak mechanical or electromagnetic (EM) impact have shown that the instability (here, slip) of the mechanical system, which is driven close to the critical state can either be initiated or hampered by strong external EM pulses (Chelidze et al., 2002, 2003). This observation, as well as earlier reports about significant influence of strong electrical impact on the dynamics of seismic systems (Tarasov et al., 1999; Chelidze and Matcharashvili, 2003) demonstrates that the EM field can affect or even control the mechanical stability of systems that are close to the critical state.

It was found experimentally that the external periodic EM or mechanical forcing leads to the clearly visible synchronization of slip in laboratory spring-slider system (Chelidze and Lursmanashvili, 2003). It was discovered that the superimposed periodic force of frequency $f$ transforms slip into periodic process with double frequency $2 f$ (it is interesting to note that earlier Chernyak, 1978, found that application to the rock sample of EM field of frequency $f$ invokes mechanical vibrations with double frequency $2 f$ ). At the same time, though the control of stick-slip by relatively weak perturbation was observed visually very clearly, to assess quantitatively the degree of correlation it was necessary to apply methods of modern synchronization theory. In the present study the phase synchronization in the experimental spring-slider system is investigated by several quantitative techniques, described in (Pikovsky et al., 2003). 


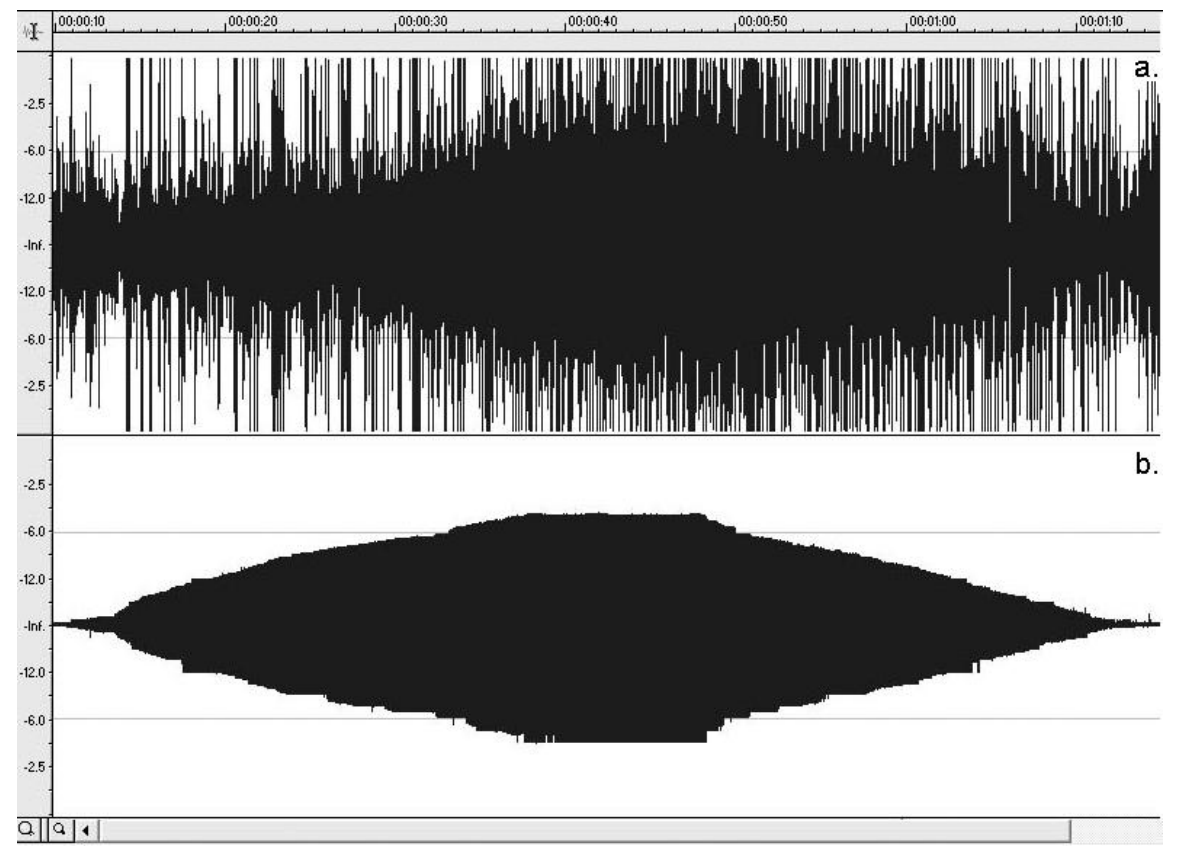

Fig. 1. Acoustic emission during slip (a) and variable external periodical EM influence (b) versus time; the amplitude of synchronizing field is given in $\mathrm{dB}$; time scale is given in microseconds. The current time $t$ is calculated based on the sampling frequency $48 \mathrm{KHz}$, which corresponds to the sampling rate once per 20 micro seconds.

\section{Methods}

\subsection{Experimental set up}

Experimental set up represents a system of two plates of roughly finished basalt (with average height of surface asperities of $0.1-0.2 \mathrm{~mm}$ ). A constant dragging force of order of $10 \mathrm{~N}$ was applied to the upper (sliding) plate weighting $0.7 \mathrm{~kg}$; in addition, the system was subjected to periodic electric $(50 \mathrm{~Hz})$ perturbations with variable amplitude (from 0 to $1000 \mathrm{~V}$ ). The effect of EM field was much weaker (of order of $1 \mathrm{~N}$ ) compared to the driving force. The electric field lines were normal to the sliding plane; that generates (ponderomotive) attraction forces between fixed and slipping plates. Slip events were recorded as acoustic emission bursts. Acoustic emission waveforms as well as the sinusoidal EM signal $(f=50 \mathrm{~Hz})$ were digitized at $48 \mathrm{kHz}$. Details of the setup and technique are given in (Chelidze and Lursmanashvili, 2003).

Here we analyze the experiment, where the superimposed periodic EM field intensity has been raised monotonously from zero to $1000 \mathrm{~V}$ and then decreased in the same way to zero (Fig. 1). Besides, we carried out more than 40 experiments with various (but constant for a given test) field intensities as well tests with step-like variation of superimposed periodic EM impact.

Different regimes of slip were excited depending on the amplitude and frequency of applied external EM field. Figure 2 illustrates the transition from typical slip synchronization effect, which is observed at voltages, exceeding 350$400 \mathrm{~V}$. The similar synchronization effects were observed, when a weak periodic mechanical force was applied parallel to the slip surface (Chelidze and Lursmanashvili, 2003). In these experiments (roughly finished basalt plates, coefficient of friction 0.47 , the spring stiffness 150 or $1000 \mathrm{~N} / \mathrm{m}$, dragging force $10 \mathrm{~N}$, humidity $=60 \%, \mathrm{~T}=25^{\circ} \mathrm{C}$ ) the superimposed mechanical force was directed parallel to the slip surface. Synchronization was observed at the amplitude of superimposed mechanical force, exceeding $0.1 \mathrm{~N}$ at $20 \mathrm{~Hz}$, or $0.5 \mathrm{~N}$ at $50 \mathrm{~Hz}$. We believe that the EM impact, which induced synchronization, is of the same order of magnitude as the mechanical synchronizing perturbation, i.e. approximately $1-10 \%$ of the dragging force.

\subsection{Quantitative methods of synchronization analysis}

In order to estimate the phases of acoustic signal we transformed digitized waveforms in order to have well pronounced sharp spikes as markers. For this purpose, after subtraction of noise, the absolute maximums of consecutive wave packages (Fig. 2) were picked up. The synchronization analysis was carried out not on the events' wave trains, shown in Fig. 2., but on the catalogue of acoustic events. More exactly the catalogue contained moments of absolute maximums picked out from the adjacent bursts separated by at least 10 consecutive zero values, one maximum per burst. The above sequence of zero values seems enough to distinguish separate bursts and, on the other side to retain in the catalogue even short bursts. In this way the catalogue, containing occurrence times and amplitudes of maximums of acoustic wave trains was compiled. Then the time series 

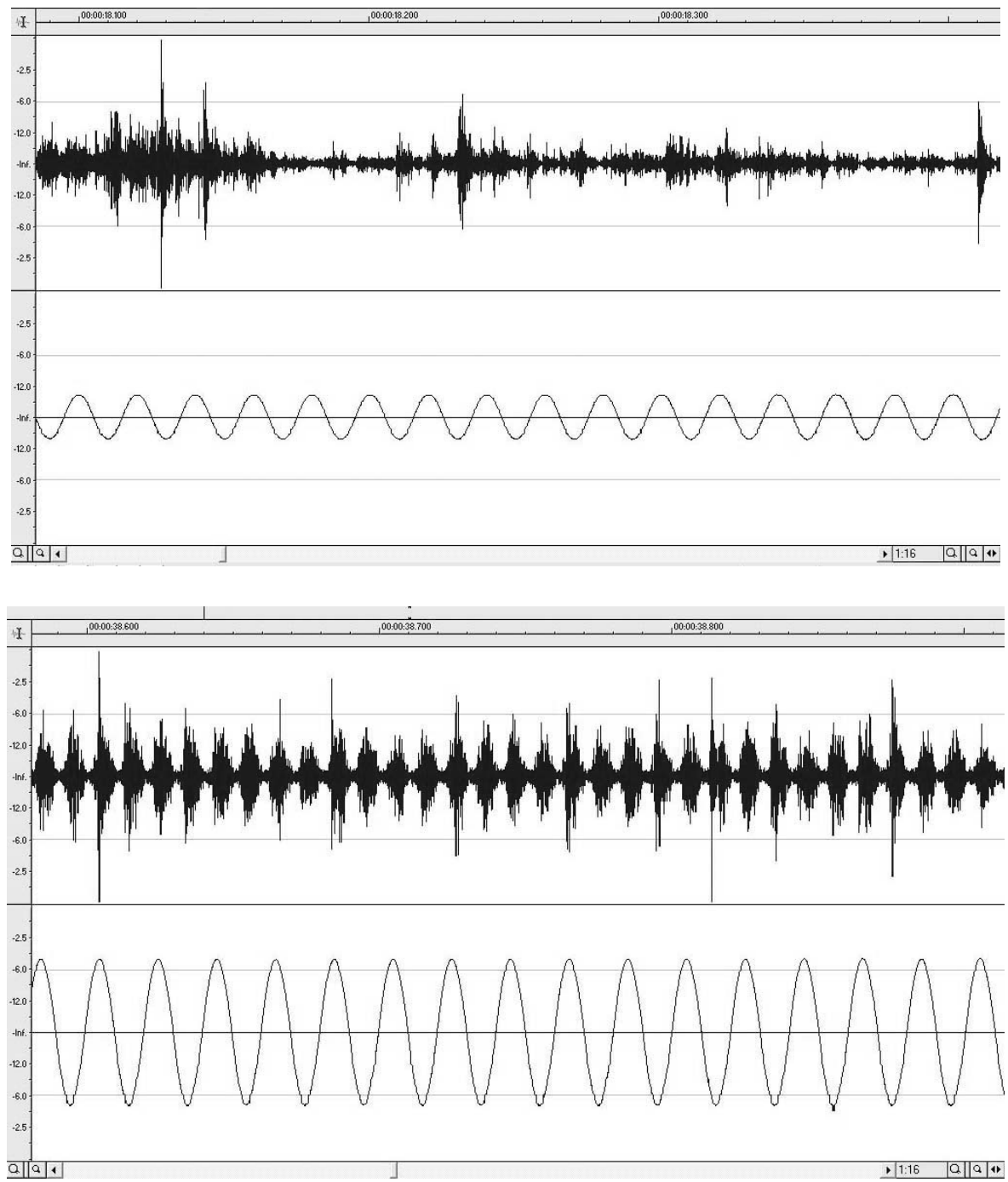

Fig. 2. Examples of completely unsynchronized (a) and synchronized (b) acoustic emissions under periodical EM influence (extended initial and central parts of the record in Fig. 1).

of time intervals between consecutive maximums (waiting times $\Delta \mathrm{t}$ ) of wave trains for $\pi$ periods of external sinusoid were composed (Fig. 3). It is evident visually that the distribution of waiting times became much narrower in the interval, where the applied field voltage exceeds approximately 500 volts.

The spectral analysis of sequence of waiting times of acoustic emissions was performed in order to confirm the effect of doubling of microslip occurrence frequency relative to that of the forcing field.

Because our dataset was transformed into a spike train, containing distinct markers, we used simple phase difference determination technique described in Pikovsky (Pikovsky et al., 2003). Additionally, in order to achieve more reliable phase construction and precise synchronization testing, the instantaneous phase of real acoustic signal was defined employing analytic signal concept, based on the Hilbert transform (Pikovsky et al., 2003). Both approaches yield similar results.
As a statistical measure of the quality of synchronization we calculated the full width at the half maximum (FWHM) of probability density distribution of phase differences. For the same reason the mean effective phase diffusion coefficient $D=\frac{d}{d t}\left[\left\langle\Delta \varphi^{2}\right\rangle-\langle\Delta \varphi\rangle^{2}\right]$ has been also calculated.

The additional quantitative tests was an assessment of the Shannon entropy

$S=-\sum_{i=1}^{N} P_{i} \log \left(P_{i}\right)$

where the probability of event to occur within the $i$-th box $P_{i}$ was calculated for the mentioned sequence of phase differences.

In order to reveal hidden details of complex dynamics of acoustic emission under external influence in the present research we used the modern method of recurrence quantification analysis (RQA). This useful tool for analysis of nonstationary relatively short data series is based on the method 


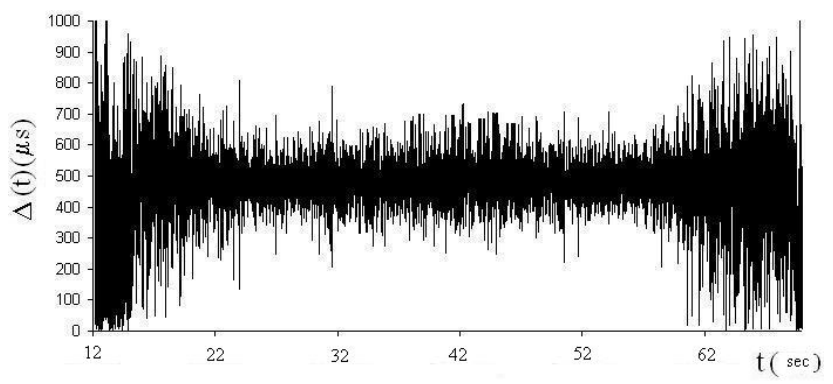

Fig. 3. Time series of time intervals between consecutive maximal amplitudes of acoustic signals (waiting times) in consecutive $\pi$-periods of external forcing.
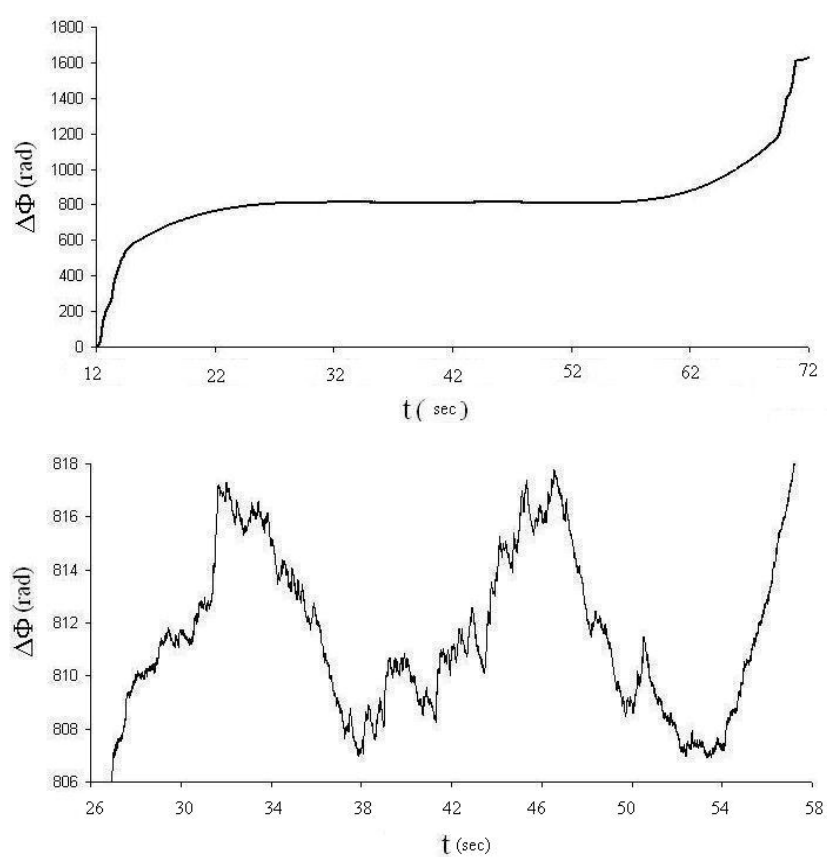

Fig. 4. (a) Phase differences between the whole sequences of maximums of acoustic emissions' bursts and external sinusoidal signal. (b) Details of phase difference plot for the most synchronized part in the $\Delta \Phi$ range between 806 and 816 .

of recurrence plots (RP), which has been introduced by Eckman (1987). According to this approach, the unknown dynamics of the phase space trajectory can be appropriately reconstructed from the time series using Takens' embedding theorem (1981).

The recurrence plot is defined as:

$R_{i, j}=\Theta\left(\varepsilon-\left\|\boldsymbol{x}_{i}-\boldsymbol{x}_{j}\right\|\right)$

here $\varepsilon$ is a cut-off distance, $\Theta(x)$ is the Heaviside function. The values one and zero in this matrix commonly are visualized as black and white points in the RP.

RQA is a quantitative extension of RP method that was introduced by Zbilut and Webber (1992). This technique defines several quantitative measures based on the analysis of diagonally oriented lines in the recurrence plot. In our research we used the recurrence rate, $R R(t)$.

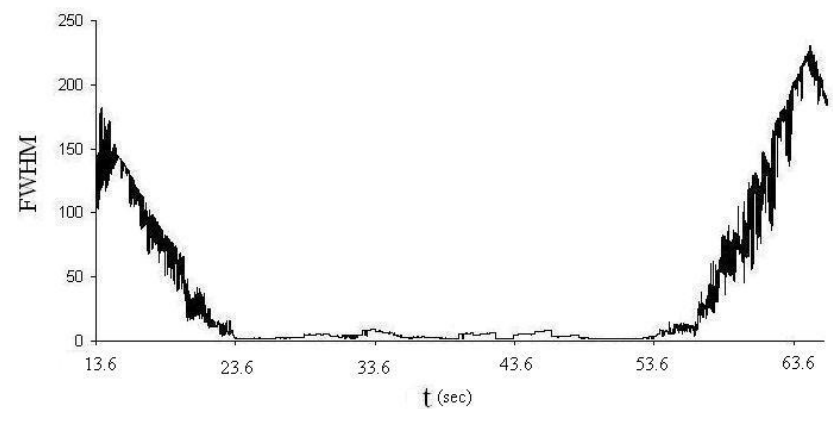

Fig. 5. Variation of the full width of probability density distribution of phase differences between the catalogue of acoustic events and periodic signal at a half maximum (FWHM), calculated for sliding windows, containing 500 consecutive events.

The recurrence rate is defined as:

$R R(t)=\frac{1}{N-t} \sum_{t=1}^{N-t} l P_{t}(l)$,

where $P_{t}(l)$ is the probability of occurrence in RP of a diagonal line of length $l, t=0, t>0$ and $t<0$ mark accordingly the main diagonal, the diagonals above and the diagonals below the main diagonal. Generally a recurrence rate is the ratio of all recurrent states (recurrence points) to all possible states of the phase space and is therefore the probability of the recurrence of a certain state. A high density of recurrence points on a diagonal results in a high value of RR. This is the case for systems, in which trajectories often visit the same section of the phase space. Therefore, random behavior of a system causes very short RP diagonals, whereas deterministic behavior causes longer diagonals.

After that, in order to investigate energetic aspect of external influence on the microslip dynamics (amplitudes of acoustic emission), we compiled time series of power of acoustic energy emitted during consecutive $2 \pi$ periods of external sinusoidal forcing. The power of acoustic emission was calculated as a ratio of the area between the acoustic signal curve and $x$-axis to the duration of $2 \pi$ period of a superimposed (experimental) sinusoidal signal (the experimental signal was not an ideal sinusiode).

Instead of the above-mentioned synchronization analysis only scaling properties of these time series were analyzed using Gutenberg-Richter type of approach.

\section{Results and discussion}

Depending on the amplitude of synchronizing force, the slip regime, recorded as acoustic emissions, varies from the perfect phase synchronization of slip events with the perturbing periodic EM impact to the complete desynchronization of microslip events and perturbations.

In Fig. 4, we report the temporal evolution of phase difference $\Delta \Phi$, obtained from Hilbert transform of waiting time sequences. Well-defined horizontal part of synchrogram (Fig. 4a) represents the time interval during which the 


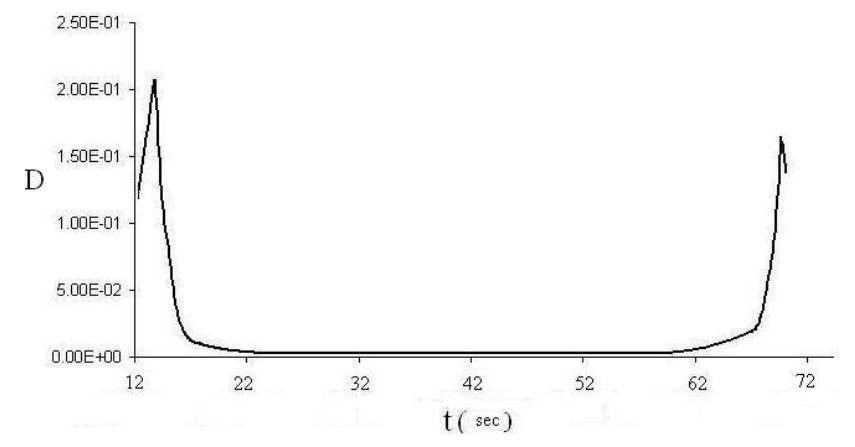

Fig. 6. Variation of phase diffusion coefficient of phase differences, calculated for sliding windows, containing 500 consecutive events.

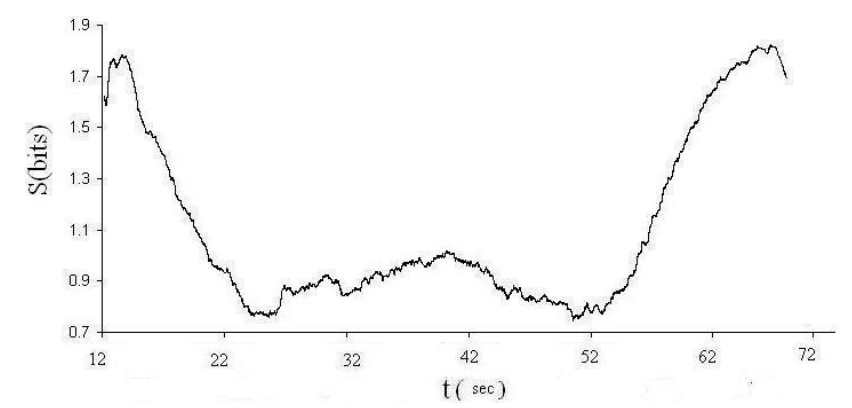

Fig. 7. Variation of Shannon entropy of phase differences, calculated for sliding windows, containing 500 consecutive events.

acoustic emissions become phase synchronized by the external sinusoidal field in the wide range of amplitudes (from approximately $500 \mathrm{~V}$ to $1000 \mathrm{~V}$ ). Phase synchronization is especially obvious in the magnified synchrogram, Fig. 4b, as long as in the most synchronized part of the plot the phase difference variation does not exceed $10 \mathrm{Rad}$, whereas outside of the mentioned domain the variation is very large and amounts to several hundreds of radians. The fine structure of the phase difference in this interval shows some long-range correlations, but at present we have not explanation of the effect.

The pdf of catalog of the phase differences between maximums in the consecutive acoustic bursts and periodic impact is narrower for the synchronized part of the catalogue compared to a non-synchronized one. That is quite natural, because we synchronize the random (or almost random) sequence of acoustic bursts by the periodic signal. Thus, the corresponding pdf should be narrow, as the absolute maximums in consecutive acoustic bursts occur almost periodically. As it follows from Fig. 5, density distribution of phase differences indeed is much narrower for the synchronized part of Fig. 1a than in sections, where the voltage is low.

Frequency locking, expressed as a minimum of phase diffusion coefficient $D$, is also a quantitative indication of the phase synchronization (see Fig. 6); it is evident that in the interval of external forcing $D$ is close to zero.

Moreover, a clear decrease of Shannon entropy value (Fig. 7) as well as the increase of percentage of recurrence

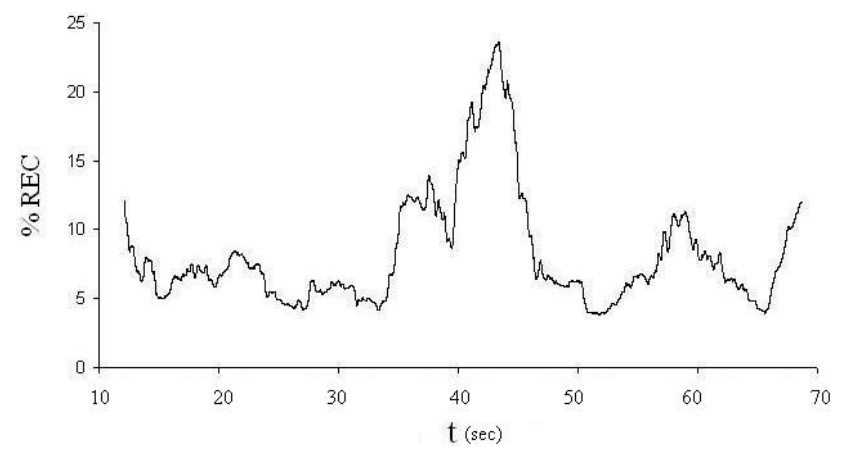

Fig. 8. Percentage of recurrence points of inter-spike time intervals sequences, calculated for sequential sliding windows, containing 400 events.

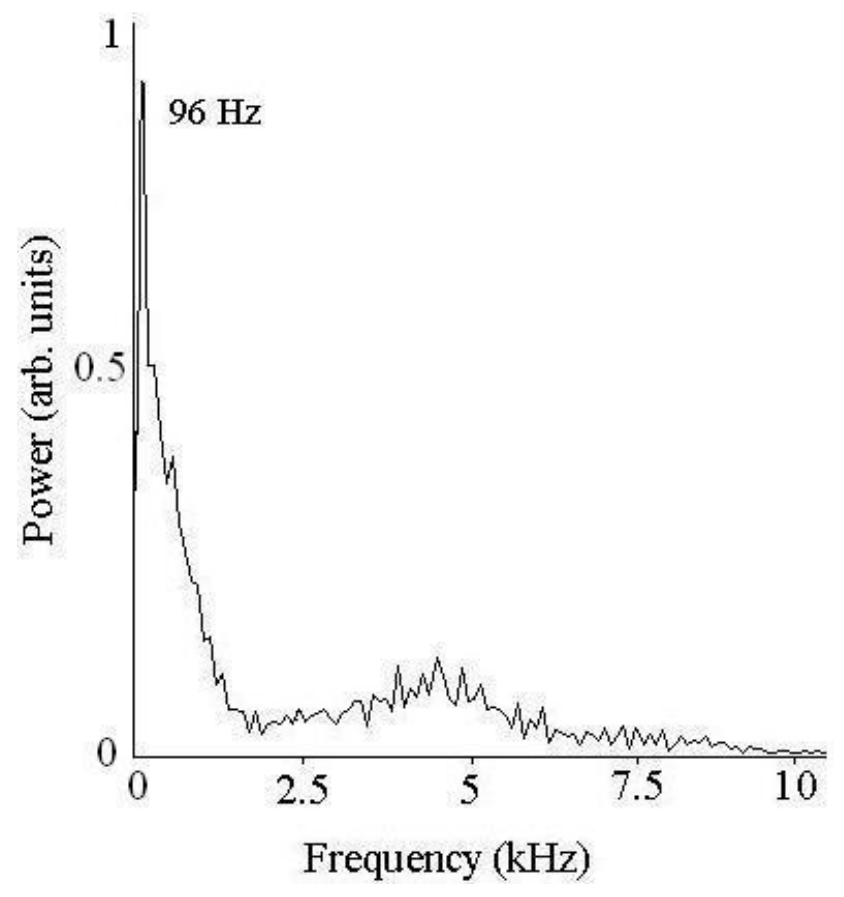

Fig. 9. Fourier spectrum of catalog of waiting times of acoustic events. Clear maximum is visible at $96 \mathrm{~Hz}$, the double frequency of the external (synchronizing) forcing.

points (Fig. 8) indicate that the dynamics of acoustic emission becomes much more regular for this part of acoustic emission data set. It is interesting that there are some transient effects before and after phase synchronization interval in acoustic emissions that are visible as two clear minima in the Shannon entropy variation (Fig. 7). We have no explanation of the effect at present.

Spectral analysis of waiting times series of analyzed quasi periodic acoustic emission process reveals global maximum at approximately $100 \mathrm{~Hz}$, which is the double frequency of forcing (Fig. 9). Mean value of inter spike intervals in waiting time series is approximately 500 micro seconds. Power spectrum of waiting time series shows $100 \mathrm{~Hz}$ main frequency. This means that the main period in mentioned 


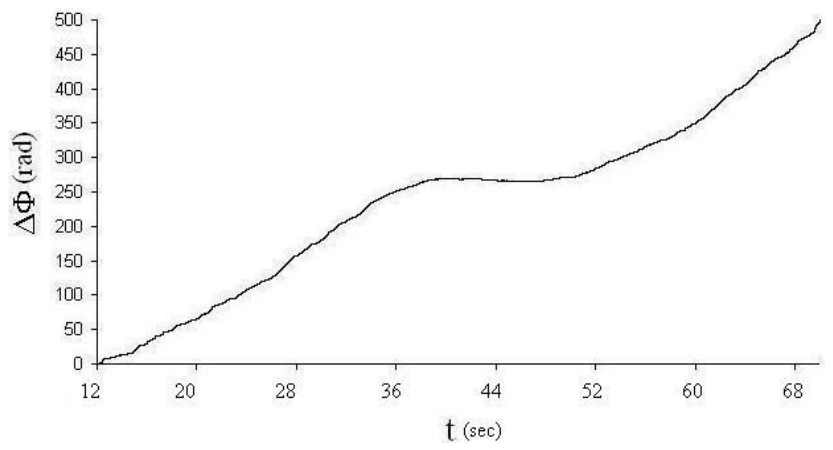

Fig. 10. Phase differences of waiting times between the power of acoustic emission and external sinusoidal signal.

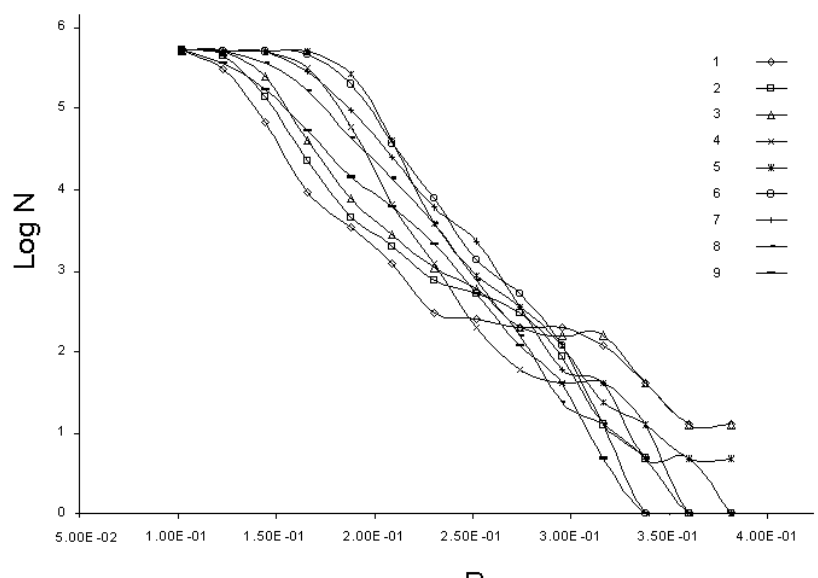

Fig. 11. Log cumulative value of number of events versus power of acoustic emission (analog of Gutenberg-Richter relation) sliding windows, containing 300 events and shifted by 300 data step. The series with numbers 1, 2, 8, 9 correspond to the time intervals, when the applied EM forcing was less than $400 \mathrm{~V}$, the series 4,5 , and 6 correspond to the maximal synchronization area (voltages 700$1000 \mathrm{~V}$ ), series 3 and 7 correspond to intermediate values of voltage.

time series with dominant 500 micro second values is about 10000 micro seconds which is quite possible for analyzed quasi periodic process with a wide distribution of waiting times (see Fig. 9). We guess that the frequency doubling could be explained by the polarization of rock plates; the polarization reaches its maximum value twice per period. One of these two polarization maximums is connected with the positive and another with the negative phase of the sinusoid. Since the electric field was oriented perpendicular to the slip surface, in both cases polarization generated the attractive force at the slip surface. That leads to the "electromagnetic braking" of slip at passing the extreme values of sinusoid (Chelidze and Lursmanashvili, 2003) and eventually, after accumulation of enough stress, to a sudden slip; as the attractive interaction does not depend on the sign of polarization, the braking occurs twice per one period of the external force. The frequency content of individual acoustic bursts lies in the range $1000-20000 \mathrm{~Hz}$ and can be related either to

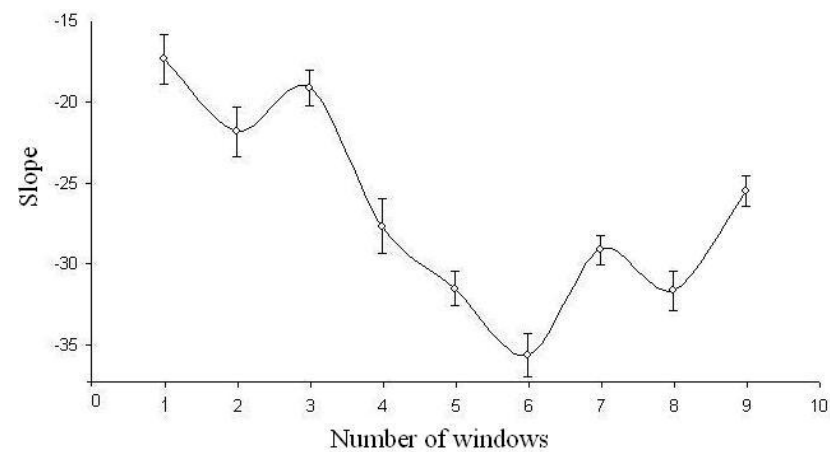

Fig. 12. Slopes of the linear parts of curves in Fig. 11 versus the number of interval (series in Fig. 11).

the properties of the slip surface or to the elastic wave generation during microslip event; the dominant frequencies are persistent in all tests irrespective of experimental conditions.

We consider our experiment as a model of natural seismic process, so it was important to see whether the power of emitted acoustic energy during $2 \pi$ cycle of external forcing may also be controlled; this is related to the nowadays very remote possibility of controlling seismic activity by external forcing (Tarasov et al., 1999). Modelling the slip energy control even on the laboratory scale seems to be very interesting. As it is shown in Fig. 10, the distribution of power of acoustic signals also reveals clear phase synchronization. What is especially important, the scaling character of energy emission undergoes changes, showing clear decrease of number of both largest and smallest events under weak periodic external influence. The analogs of Gutenberg-Richter relationships were plotted for nine different time intervals using sliding windows containing 300 events shifted by 300 data steps. The series with numbers 1, 2, 8, 9 correspond to the time intervals, when the applied EM forcing was less than $400 \mathrm{~V}$, the series 4,5 , and 6 correspond to the maximal synchronization domain (voltages 700-1000 V), series 3 and 7 correspond to intermediate values of voltage (Fig. 1).

It follows from Figs. 11 and 12 that the slope of Gutenberg-Richter relationship significantly increases under synchronization. That means that the energy is pumped from extremely small and very large events to some intermediate scale; this also means that in principle it is possible to regulate the amount of emitted energy per event using external synchronization technique at least in the laboratory conditions. Realization of similar "quantization" approach in the field conditions for releasing accumulated elastic energy in relatively small portions is, of course, improbable at present, but cannot be excluded in the future.

The above experiment was performed for a varying intensity of forcing at a single forcing frequency of $50 \mathrm{~Hz}$. At the same time it is known that the synchronization strength depends not only on the intensity, but also on the frequency of the external forcing. That is why the similar experiments were carried out for different frequencies also. The results are presented on the Fig. 13. It is evident that the 
synchronization field manifests a typical configuration that is known as the Arnold's tongue (Pikovsky et al., 2003); for a given intensity of forcing synchronization fades at very low and very high frequencies. There is an optimal frequency (here $60 \mathrm{~Hz}$ ), where the synchronization is achieved at the minimal forcing (here $\sim 400 \mathrm{~V}$ ). On the borders of Arnold's tongue the synchronization is intermittent. The preliminary experiments show that the location of Arnold's tongue depends on the physical characteristics of slider-spring system, such as a mass of the sliding plate (normal stress), stiffness of spring, roughness of plate surfaces, etc. For example, the EM synchronization effect for the well-finished glass plates (for the sliding plate, weighting $\sim 100 \mathrm{~g}$ ) was observed at the forcing intensity $1000 \mathrm{~V}$ at the frequency $1000 \mathrm{~Hz}$.

Beeler and Lockner (2003), in following BL, carried out the laboratory experiments on rock friction, when the small (mechanical) sinusoidal loading is added to the linear shearing load. They mapped the frequencies and amplitudes of superimposed loading that causes correlation of slips with sinusoidal signal, i.e. they compiled the analog of Arnold's tongue. Beeler and Lockner introduce some characteristic time $t_{c}$ and distinguish two regimes: the first one corresponds to large periods $T\left(T>t_{c}\right)$, where the amplitude of sinusoidal loading $A$, necessary for correlation, decreases with frequency $f$ and the second one for $\left(T<t_{c}\right)$, where $A$ is independent of $f$. The critical time they relate to the duration of failure (slip) nucleation phase. Our experiments, presented in this paper (TP) differ from these of Beeler and Lockner in several aspects: i. BL applied to the samples high confining pressure $50 \mathrm{MPa}$. In TP the normal stress is low and is actually realized by the weight of the sliding sample $(700 \mathrm{~g})$; ii. In BL the synchronizing force is mechanical and in TP it is electromagnetic; iii. In BL the synchronizing frequencies are very low, from 0.001 to $1 \mathrm{~Hz}$. In TP the optimal synchronization (minimal synchronizing force) is achieved at the frequency $60 \mathrm{~Hz}$. Of course, the difference is explained by different conditions of experiment, especially by the differences in the normal stress; iv. In BL the number of slip events in catalogues at each combination of loading rate, frequency and amplitude was around 20, in our experiments the catalogues contain several thousand events for each test. Large statistics, obtained in our experiments allows application of many methods of quantitative nonlinear analysis, which is impossible with catalog, containing 20 events.

It seems that there is nevertheless some similarity in our results, namely, the observation of Arnold's tongues, which in $\mathrm{BL}$ is discussed as a transition from the negative to positive response mode at $T<t_{c}$. By the way, BL theoretical model predicts frequency independence of $\mathrm{A}$ in the high frequency domain, but their experiments demonstrate clearly the systematic increase of $\mathrm{A}$ at high frequencies, which if compatible with universal Arnold model of synchronization. Of course, the minimum of Arnold's tongue in BL and TP is observed at different frequency and amplitude intervals, due to the big difference in the conditions of experiments. It should be noted that the formalism of synchronization theory do not provide the physical mechanism of underlying pro-

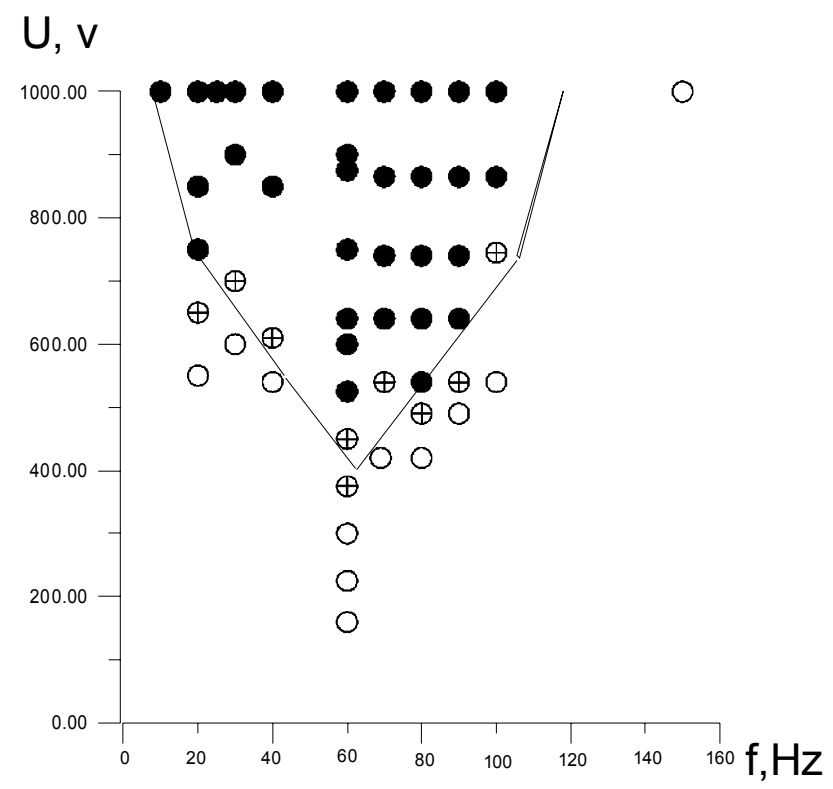

Fig. 13. Stick-slip synchronization area (Arnold's tongue), corresponding to various intensities and frequencies of the external forcing. On the $y$-axis the voltage and on the $\mathrm{x}$-axis the frequency of the applied periodical EM field are plotted. Filled circles correspond to the perfect phase synchronization (PS) and empty ones correspond to the absence of PS. Circles with crosses mark the areas of intermittent PS.

cesses. The physics of processes can be revealed only by series of critical experiments carried out in various conditions.

The interesting consequence of the research is that one has to investigate a wide range of frequencies in order to find the optimal synchronizing frequency of the Arnold's tongue. According to Beeler and Lockner (2003) the characteristic time of earthquake preparation process is of order of years. Correspondingly, in the case of earthquakes the optimal synchronizing force could be connected with the long-term components of gravity force spectrum with semi-annual and annual periods (Crossley et al., 1999). That can also explain the negative conclusion of Vidale et al. (1998) and Beeler and Lockner (2003) on a very weak correlation of seismicity with earth tides of semi-diurnal and diurnal periods, as their duration is much less that the characteristic time of seismic process.

\section{Conclusions}

Application of a weak external field can significantly affect the slip regime of spring-slider system. External periodic electromagnetic forcing leads to a clear phase synchronization of microslip events in a wide range of amplitudes. The quality of phase synchronization was assessed by several quantitative methods of analysis. The synchronization field, plotted in terms of synchronizing force versus its frequency, manifests a typical configuration that is known as the Arnold's tongue. The energy release in the stick-slip process 
is also affected by synchronization: the scaling character of energy emission undergoes changes, showing clear decrease of number of both largest and smallest events under weak periodic external forcing.

Acknowledgements. This work was supported by INTAS grant: $\# 0748,2002$. Authors are extremely grateful to (anonymous) referee for constructive remarks, which contribute much to the paper.

Edited by: C. Goltz

Reviewed by: three referees

\section{References}

Beeler, N. M. and Lockner, D. A.: Why earthquakes correlate weakly with the solid Earth tides: Effects of periodic stress on the rate and probability of earthquake occurrence, J. Geophys. Res., B108, 2391-2405, 2003.

Chelidze, T. and Lursmanashvili, O.: Electromagnetic and mechanical control of slip: laboratory experiments with slider system, Nonlin. Proc. Geophys., 20, 1-8, 2003.

Chelidze, T. and Matcharashvili, T.: Electromagnetic control of earthquakes dynamics?, Computers and Geosciences, 29, 587593, 2003.

Chelidze, T., Varamashvili, N., Devidze, M., Tchelidze, Z., Chikhladze, V., and Matcharashvili, T.: Laboratory Study of Electromagnetic Initiation of Slip, Ann. Geophys., 45, 587-599, 2002.

Chernyak, G.: On the physical nature of seismoelectric effect in rocks, Izvestia Ac. Sci. USSR, Physics of Earth, in Russian, N2, 108-112, 1978.

Crossley, D., Hindered, J., Casula, G., et al.: Network of Superconducting Gravimeters Benefits a Number of Disciplines, EOS Transactions, AGU, 80, 11, 121-126, 1999.

Custodio, S., Fonseca, J., Faria, B., and d'Oreye, N.: Tidal modulation of volcanic tremor in Fogo Island, Cape Verde, Book of Abstracts, European Seismological Commission XXVIII Assembly, Genoa, 236, 2002.

Dieterich, J.: Nucleation and triggering of earthquake slip: Effect of periodic stresses, Tectonophysics, 144, 127-139, 1987.

Eckmann, J. P., Kamphorst, S. O., and Ruelle, D.: Recurrence plots of dynamical systems, Europhys. Lett., 4, 973-977, 1987.
Grasso, J.-R.: Mechanics of Seismic Instabilities Induced by the Recovery of Hydrocarbons, Pageoph, 139, 507-534, 1992.

Matcharashvili, T., Chelidze, T., and Javakhishvil, Z.: Nonlinear analysis of magnitude and interevent time interval sequences for earthquakes of the Caucasian region, Nonlin. Proc. Geophys., 7, 9-19, 2000,

SRef-ID: 1607-7946/npg/2000-7-9.

Nikolaev, A. V. (Ed.): Induced Seismicity, Moscow, "Nauka", in Russian, 220, 1994.

Perfettini, H. and Schmittbuhl, J.: Periodic loading on a creeping fault: Implications for tides, Geophys. Res. Lett., 28, 435-438, 2001.

Pikovsky, A., Rosenblum, M. G., and Kurth, J.: Synchronization: Universal Concept in Nonlinear Science, Cambridge University Press, Cambridge, 411, 2003.

Rosenblum, M. G., Pikovsky, A., and Kurth, J.: Phase synchronization of chaotic oscillators, Phys. Rev. Lett., 76, 1804-1808, 1996.

Rosenblum, M. G., Pikovsky, A., and Kurth, J.: Effect of phase synchronization in driven chaotic oscillators, IEEE Trans. CASI, 44, 874-881, 1997.

Rundle, J., Turcotte, D., and Klein, W. (Eds.).: Geocomplexity and the Physics of Earthquakes, Geophys. Monograph 120, American Geophysical Union, Washington, DC, 284, 2000.

Scholz, C. H.: Good tidings, Nature, 425, 670-671, 2003.

Sobolev, G. A. and Ponomarev, A. V.: Physics of Earthquakes and Precursors, Moscow, "Nauka", in Russian, 270, 2003.

Takens, F.: Detecting strange attractors in turbulence, In: Dynamical Systems and Turbulence, Warwick 1980, edited by: Rand, D. A. and Young, L. S., 898, Springer Lecture Notes in Mathematics, Springer, Berlin, 366-381, 1981.

Tarasov, N. G., Tarasova, N. V., Avagimov, A. A., and Zeigarnik, V. A.: The effect of high-power electromagnetic pulses on the seismicity of the central Asia and Kazakhstan, Vulkanologia i seismologia, in Russian, 4-5, 152-160, 1999.

Vidale, J., Agnew, D., Johnston, M., and Oppenheimer, D.: Absence of earthquake correlation with earth tides: an indication of high preseismic fault stress rate, J. Geophys. Res., 103, $24567-$ 24 572, 1998.

Wang, Y., Mora, P., Yin, C., and Place, D.: Statistical tests of loadunload response ratio signals by lattice solid model, Pure Appl. Geophys., 161, 1829-1839, 2004.

Zbilut, J. P. and Webber Jr., C. L.: Embeddings and delays as derived from quantification of recurrence plots, Physics Letters A, 171, 199-203, 1992. 